\title{
Minor procedures in the accident and emergency department: can Entonox help?
}

\author{
C. G. LEESON PAYNE*, D. L. EDBROOKE* \& \\ G. K. DAVIES ${ }^{+}$ \\ * Department of Anaesthetics, Royal Hallamshire Hospital, Sheffield and \\ ${ }^{+}$Department of Anaesthetics, Northern General Hospital, Sheffield
}

\section{SUMMARY}

The efficacy of Entonox as a supplement to local anaesthesia for minor surgicain procedures was studied. Eighty-five patients undergoing surgery for the incision and drainage of a subcutaneous abscess using a local anaesthetic were involved in a trial to ascertain the level of pain associated with such procedures and to investigate the possibility of using on-demand Entonox to supplement the locals anaesthesia provided. Patients received either the standard local anaesthesia, or, $\overrightarrow{0}$ alternatively, the local anaesthesia was supplemented with oxygen or Entonox voa? on-demand apparatus.

Patients indicated on three linear visual analogue scales their pain, anxiety a total discomfort, and also on a cartoon pain rating scale, at different stages during the operation.

Results showed that there was no statistically significant reduction in pain $\stackrel{\infty}{\circ}$ provided by the Entonox, despite general patient approval. Both oxygen ando Entonox showed some anxiolytic properties. Pure oxygen was also seen to produce a rise in heart rate, while both gases (pure oxygen and Entonox) caused ano increase in diastolic blood pressure. It was also seen that the patient's short termo memory of the pain experienced was unaffected by pure oxygen or Entonox.

\section{INTRODUCTION}

Pain and anxiety are common in any accident and emergency department. For many people it is their first and sometimes only contact with a hospital as ao patient. Despite the relative triviality of many medical complaints, the necessityr

Correspondence: $\operatorname{Dr}$ C. G. Leeson Payne, Department of Anaesthetics, G Floor, Royal Hallamshire Hospital, Glossop Road, Sheffield S10 2GJ, U.K. 
for even minor surgical intervention to an otherwise perfectly healthy individual can seem like a major crisis.

In this situation, impressions and experiences, particularly of pain, can last a lifetime in the patient's memory. Every effort should be made to decrease the psychological and physical stress of all forms of minor surgery carried out under such circumstances. The primary factor in attending to patient discomfort must be awareness of the problems by all medical staff. This awareness may be lacking when speed is a driving force and excess familiarity its advocate. With this apparent absence of concern goes a paucity of comprehensive research, although Melzack \& Wall (1982) investigated acute pain in an emergency clinic across a range of presenting conditions. The pain associated with minor operative procedures, has not been the subject of extensive investigations.

A discrete group of painful conditions that provides a common background for investigation are those patients requiring incision and drainage of soft tissue abscesses, many of which are carried out using local anaesthetic techniques alone. It is highly likely that this method of anaesthesia is inadequate and requires supplementation by other means.

The use of Entonox ( $50 \%$ nitrous oxide/50\% oxygen) in addition to the local anaesthetic may make a substantial difference to the pain relief already provided. Entonox has been used for many years, particularly on an on-demand basis for the reduction of pain in labour. More recently, Entonox has been proved useful in dental care (Hallonsten, 1983) and when administered to injured patients prior to hospital admission. (McKinnon, 1981).

The purpose of this study was to analyse the pain of the peri-operative period by using linear visual analogue scales and to relate these findings to the anxiety experienced and to a pain/anxiety combination factor known as 'total discomfort', both measured in a similar way. A cartoon subjective scale, analogous to an adjectival scale (Melzack \& Torgerson, 1971), was also used by the patients, as it is suggested that pain pictures may be as appropriate to adults as to children (Unruh, 1983). In addition, the suitability and efficacy of Entonox as a supplemental analgesic was assessed.

\section{SUBJECTS AND METHOD}

Fit patients between 16 and 60 years old who had presented at the Accident and Emergency Department of Royal Hallamshire Hospital, Sheffield needing a local anaesthetic (rather than a general anaesthetic), as deemed by the casualty officer, for the incision and drainage of a soft tissue abscess, were introduced to the trial, $30 \mathrm{~min}$ before the minor procedure operating list. Any patients having an infected foreign body requiring removal, or any having taken alcohol or any analgesic or antibiotic medication or being in receipt of any treatment, prescribed or self administered, prior to admission were excluded from the trial. On signing an informed consent form, the patients were sequentially allocated to one of three groups: 
(1) Group N, comprising those patients who received only the standard local anaesthesia for the procedure.

(2) Group O, comprising those who were to have the local anaesthesia supplemented by oxygen as supplied on an on-demand basis.

(3) Group E, comprising those who were to have the local anaesthesia supplemented by Entonox as supplied on an on-demand basis.

During the course of the study it became evident that there was a significant group of patients in the trial who refused to have the additional analgesia provided by the 'gas' in whatever form. The data concerning these patients was still recorded.

As the 'normal' procedure in A\&E does not involve the use of Entonox, the patients were not told in advance whether they fell into group $\mathrm{N}, \mathrm{O}$ or $\mathrm{E}$.

However, those in group $\mathrm{E}$ or $\mathrm{O}$ were given instruction in the use of the ondemand gas supply apparatus before the local anaesthetic was given. A consistent approach to all patients by only one researcher and standard collection times for the data was used to maximize validity. The following data were collected by interviewing the patient:

Subjective data pain; anxiety; and total discomfort

These were presented to the patients as three linear visual analogue scales in the form of a modified abacus where the patient moved a single bead along each of three $20 \mathrm{~cm}$ unmarked bars, from where apointer indicated a particular numerical value on the back of the scale (unseen by the patient) for statistical analysis.

Cartoon pain scale (Fig 1). A series of five cartoons drawn to represent degrees of pain by facial expression to relate to the patient's pain.

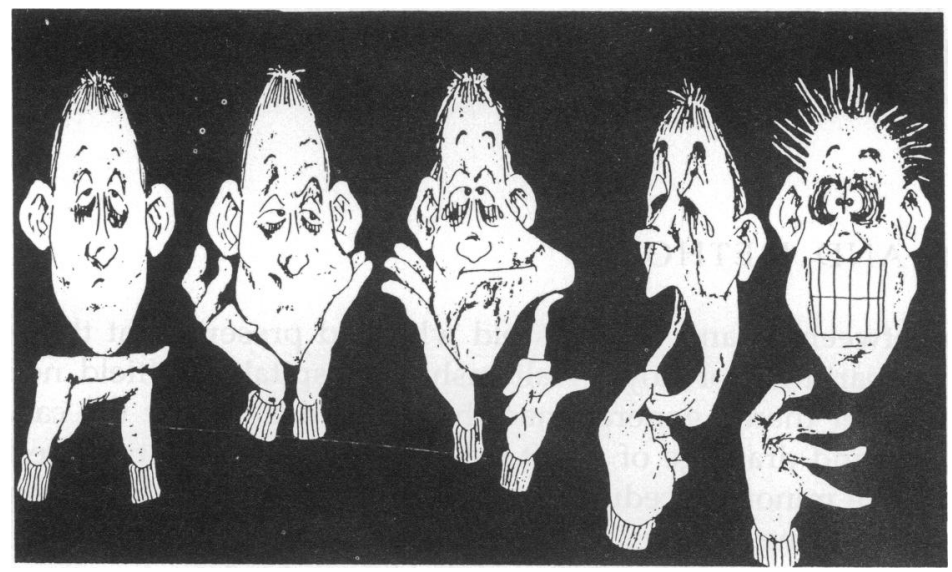

Fig 1. Cartoon pain scale. 
Objective data. Heart Rate; Systolic and Diastolic Blood Pressure

Measured using a Hitachi HME-20 automatic pulse and blood pressure monitor. This series of seven values was collected at the following times peri-operatively:

(1) Pre-operatively - (Before any mention of the use of the on-demand apparatus was made to patients in groups $\mathrm{O}$ oe $\mathrm{E}$ ) to provide data zero point values for all the groups.

(2) Immediately after the first incision into the abscess - following the patient's worst peri-operative time.

(3) Five min following the application of the surface dressing, to allow sufficient time for the Entonox to wear off. The subjective scales were marked to refer to the experiences remembered from the procedure from time (2).

(4) Fifteen min following the application of the surface dressing, prior to discharge.

The patients in groups $\mathrm{O}$ and $\mathrm{E}$, having accepted the use of the on-demand gas, although blind as to whether it was oxygen or Entonox, inhaled via the apparatus for a full minute prior to the local anesthetic administration and continued with the 'gas' until it was not longer thought necessary, usually just prior to the application of the surface dressing. There was also a short respite from using the apparatus between the local anaesthetic injection and beginning the incision. Suitable warning, a minute before the first incision was made was given to begin using the on-demand apparatus for those in groups $\mathrm{O}$ or $\mathrm{E}$. Identically timed warnings were also given to those patients in group $\mathrm{N}$ or to those who had refused administration of the gas.

The on-demand gases were supplied to the patients via the standard B.O.C. (Ohmeda) Entonox on-demand valve attached to an F size Entonox cylinder or an E size oxygen cylinder, and a length of black anti-static corrugated plastic tubing and a clear perspex face mask incorporating an expiratory flap valve. Pure oxygen was used as a placebo gas for two reasons. Firstly, Medical Air was at the time not available in pin-indexed cylinders, and secondly, the Entonox valve required only minor modification to fit an oxygen cylinder. It was completely safe in this respect since the valve is built to satisfactorily regulate pure oxygen in the event of nitrous oxide and oxygen becoming separated in the Entonox cylinder at low temperatures.

Table 1. Patient groups.

\begin{tabular}{lc}
\hline Groups & $n$ \\
\hline $\mathrm{N}$ & 23 \\
$\mathrm{O}$ & 24 \\
$\mathrm{E}$ & 23 \\
Gas Refusers & $\underline{15^{*}}$ \\
Total & $85^{\dagger}$ \\
\hline${ }^{*}$ Male $=5$, female $=10 .^{+}$Male $=$ \\
60, female $=25$
\end{tabular}


Table 2. Operation sites and group distribution.

\begin{tabular}{lrrrrr}
\hline Operation sites & Total & N & O & E & Gas Refusers \\
\hline R Infected Ingrowing Toenail & 26 & 8 & 8 & 4 & 6 \\
R Finger Paronychia & 30 & 8 & 8 & 9 & 5 \\
I Post. Auricular Infected Cyst & 8 & 3 & 2 & 2 & 1 \\
I Shoulder Abscess & 3 & 1 & 1 & 1 & 0 \\
I Abscess in Middle of Back & 1 & 0 & 0 & 1 & 0 \\
I Infected Sebaceous Cyst & 6 & 1 & 2 & 2 & 1 \\
I Scalp Abscess & 2 & 1 & 0 & 1 & 0 \\
I Toe Abscess & 1 & 0 & 1 & 0 & 0 \\
I Maxillary Abscess & 1 & 1 & 0 & 0 & 0 \\
I Tibial Abscess & 1 & 0 & 0 & 1 & 0 \\
I Natal Cleft Abscess & 1 & 0 & 0 & 1 & 0 \\
R Finger Pulp Space Abscess & 4 & 0 & 1 & 1 & 2 \\
I Axillary Abscess & 1 & 0 & 1 & 0 & 0 \\
Totals & 85 & 23 & 24 & 23 & 15 \\
\hline
\end{tabular}

$\mathrm{R}=$ Ring Blocks

$\mathrm{I}=$ Infiltration Blocks

In every case, $2 \%$ Lignocaine plain was used as the local anaesthetic, introduced via a 23 or 25 gauge needle.

\section{RESULTS}

Non-parametric statistical analysis was used throughout due to the nature of the subjective scales.

(i) The Kruskal-Wallis One Way Analysis of Variance was used initially to find out if one of the treatment groups $(\mathrm{N}, \mathrm{O}$ or $\mathrm{E})$ had a different profile over the perioperative period from the other two, for a particular parameter (pain or anxiety for example). If an odd profile was found, a Wilcoxon Rank Sum Test for unpaired data on the specific parameters, was used to identify which treatment group was the odd one out. By comparison with graphs plotted to show the mean values (merely as a guide) (graphs 1 to 4 for example see Figure 2) and their variation from the pre-operative data zero position over the course of the operative procedure, the following statements can be made:

(a) Patients in the Oxygen group experienced a significant rise in their heart rate

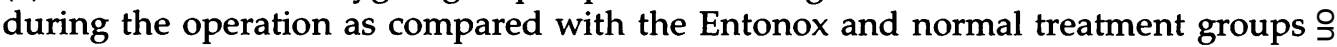
$(P<0.05)$.

(b) Patients in the oxygen and Entonox treatment groups experienced a significant rise in diastolic blood pressure when compared with the normal treatment $\stackrel{N}{\circ}$ group $(P<0.05)$.

(c) Oxygen and Entonox groups showed a significant reduction in their anxiety levels compared with the normal treatment group, $(P<0.05)$ but the difference $\bar{Q}$ in the effect between the two gases is negligible.

(d) There was no statistically significant difference in the pain experienced by any of the patient groups. 
GRAPH1.

PAIN LIUEAR VISUAL ANALOGUE YARIATJON.

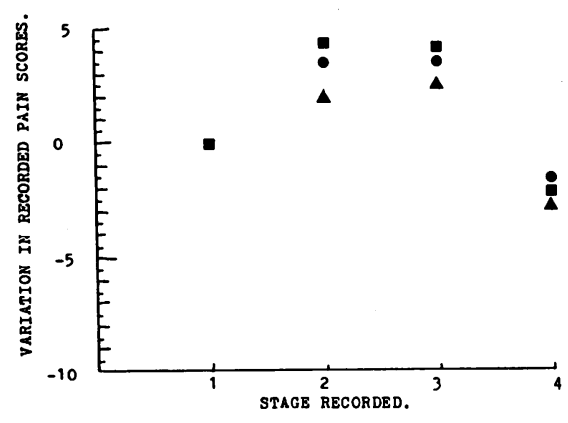

GRAPH3.

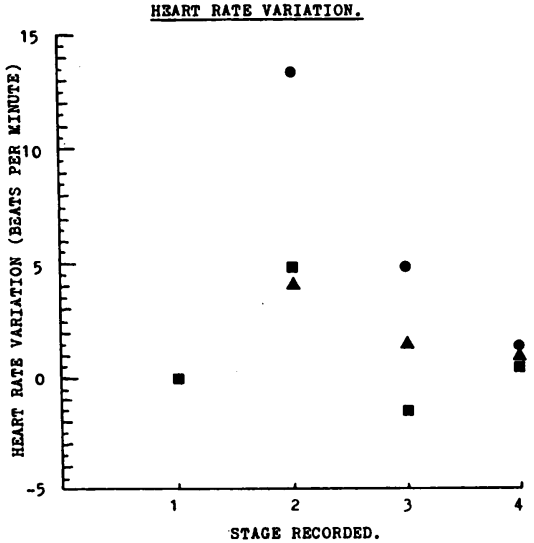
GRAPH2.

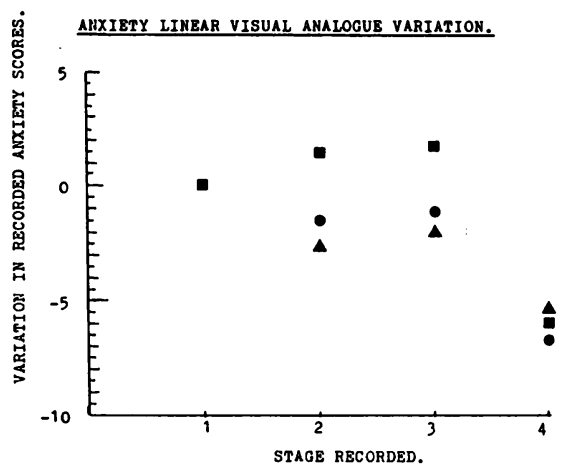

GRAPH4.

DIASTOLIC BLOOD PRESSURE VARIATION,

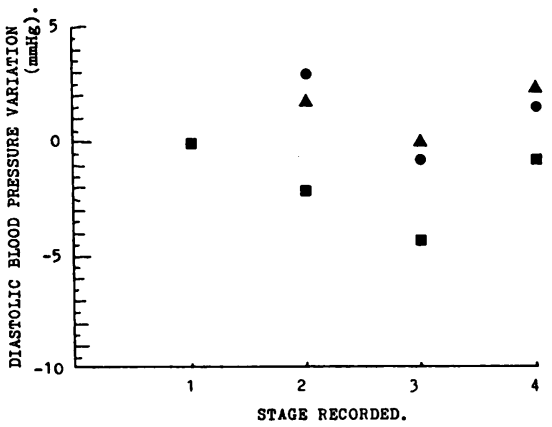

Fig 2. Each graph shows the data zero point for each group (N, O or $\mathrm{E})$ as a single point at stage 1 (pre-operatively). Key: Normal group $\square$ Oxygen group • Entonox group $\bullet$

(ii) The patients' short term memory of their worst experience of the operative procedure was analysed by comparing each patient's recording of pain, anxiety and total discomfort taken at time (2) and time (3) (see Subjects and Method). The Wilcoxon Rank Sum Test for paired data showed the patients' short term recollection was very accurate, regardless of the additional inhalational anaesthesia.

\section{DISCUSSION}

The intricacies of the appreciation of human sensation have never been easy to quantify. A controlled clinical trial using real patients rather than volunteers in the research of analgesia is difficult.

Despite the fact that Entonox appears to be a very popular analgesic agent and 
has even been described in situations very similar to those studied here, the comparison of its use against control groups is rare. Maloney et al., (1981) and $\stackrel{\$}{\$}$ Flomembaum et al., (1979) commented favourably on the use of nitrous oxide/ oxygen mixtures as a supplemental analgesic for minor dermatological surgery, $\stackrel{\mathbb{\mathrm { d }}}{\mathrm{a}}$ resulting from patient questioning, but could only conclude that nitrous oxide provided analgesia by a few patients' memory of their last similar experience. Parbrook \& Kennedy (1964) did a double blind trial of various nitrous oxide/등 oxygen concentrations in the post operative pain situations and found nitrous $\frac{\bar{m}}{\bar{n}}$ oxide gave a significant improvement in that particular situation.

To compare the authors' trial with the two American trials (Flomembaum et al., \& 1979; Maloney et al., 1980) it is notable that $100 \%$ of the Entonox-using patients $\vec{\circ}$ said the Entonox had been of benefit to them (better than the previous research) yet gave linear visual analogue readings that were no different from either of the $\vec{\omega}$ control groups.

But why should an almost universally agent be of so little apparent tangible $\underset{\infty}{\frac{3}{3}}$. benefit in this situation, as shown by this trial? The pain felt during a minor:procedure is very much a 'fast' or pin prick type of pain which, although quite ${ }_{\perp}^{+}$ impressively painful, is very short lived and barely outlasts the original noxious? stimulus.

Although the stimulus may be repeated, by virtue of its intensity and duration, its effects are mainly transmitted by the fast conducting A delta fibres rather than $\overline{\mathcal{S}}$ the slower conducting unmyelinated $\mathrm{C}$ fibres. By noting the situations in which $\overrightarrow{0}$ Entonox is currently used, it can be seen that nitrous oxide is much better gt $\stackrel{\bullet}{.}$ reducing the pain of 'slow' transmission or 'second' pain. In situations whe muscle spasm is prominent (for instance during labour or following shoulde⿳⺈, dislocation) it appears to be particularly useful in pain reduction.

Entonox here has been statistically shown to produce no significant improvement $\frac{\partial}{D}$ in analgesia, but it is seen to produce an effective means by which anxiety can be $\stackrel{\varrho}{\vec{D}}$ reduced during the operation. Its effect however, is no different from that demonstrated by oxygen alone. Whether this is totally a placebo effect or the oxygen contributing to the anxiolytic effect is unclear.

The ineffectiveness of nitrous oxide as an analgesic agent in this study provides added evidence about the difference between pain in clinical situations and that created in the laboratory using volunteers. Dworkin et al., (1983) showed quantifi- $\underline{3}$. able, statistically significant, and subjectively meaningful increases in thresholds $\delta$ for sensation and pain, and pain tolerance with nitrous oxide, of concentrations of 3 $15-45 \%$ percent, in volunteers. Evidently, the anxiety of the real clinical situation? enhances the feelings of pain and reduces the efficacy of nitrous oxide at this sub- $-\frac{7}{O}$ anaesthetic concentration, even though using an on-demand supply of gas (oxygen or Entonox) does reduce anxiety. This feature cannot be measured in the exper- $\stackrel{\sigma}{\sigma}$ imental situation. It is also clear that the added concern of using the on-demand $N_{0}$ apparatus does not increase the patient's anxiety, although the rise in diastolic $\mathbb{N}^{\mathbb{N}}$ blood pressure may be an objective reflection of this anxiety.

With very few exceptions all the patients stated that the pain relief provided ${ }^{\circ}$ during the procedure had been sufficient. From this assessment alone it is possible to say that the analgesia provided by a simple local anaesthetic block is perfectly 
adequate in most situations for this type of minor procedure. However, there are a few notable exceptions which depend on the site of the abscess on the body, that requires very careful judgment before proceding with a local anaesthetic procedure alone. These include natal cleft abscesses and any other large abscess that cannot utilize a ring block for their anaesthesia.

The linear visual analogue was, once again, demonstrated as being a readily acceptable and relevant means of measuring sensation. It suffers no more than any of the other pain scales in the arguments about the ordinance and magnitude of its readings. Measuring the complementary references of pain and anxiety together, the efficacy of analgesia can be investigated more completely in the clinical situation. The concept of total discomfort is perhaps more difficult to appreciate, but it does represent a slightly different angle on a common situation.

The cartoon pain scale on the other hand, offers rather different problems. Despite the effort to make the communication of pain on a more human level, we have made the situation even more complex. There is no human expression that can reflect a singular pure emotion except in its most extreme manifestation, and yet the cartoon pain scale attempted just that. Although the pain scale was instantly and universally popular; patients found it difficult to choose which one best reflected their pain. Had an order of the cartoons (from no pain to severe pain) not been suggested, some very haphazard observations would have been made. All the patients found the cartoons much more difficult to interpret that the rather inhuman linear visual analogue scales; rather surprisingly, since the cognitive appreciation of pain is rarely visualized as a point on a line, but more often as an abstract perception or at least a series of words.

The changes seen during the administration of Entonox of the physiological parameters is yet another indicator of the differences between the laboratory and the operating theatre. Whilst breathing a gas in the unstressed situation does not produce changes in blood pressure and heart rate, breathing the same gas in an anxious situation, especially having been conditioned to believe that the more of the gas they breathed, the less pain will be experienced, it is hardly surprising that a completely different impression is created. The significant rise in heart rate associated with the oxygen treatment group and the rise in diastolic blood pressure associated with both gas treatment groups demonstrates this phenomenon.

The possibility of hyperventilation tells a different story from Dottori et al., (1976) when they said that measurements of cardiovascular function do not change to a clinically significant degree during induction of analgesia with nitrous oxide, and that any changes that did occur paralleled the use of $100 \%$ oxygen (Everett et al., 1971; Triger et al., 1971).

The cardiorespiratory depressant actions attributed to nitrous oxide (Sonnerstein et al., 1948; Parbrook, 1967) may be partly counteracted by the high oxygen concentration in the Entonox to provide effects on the diastolic blood pressure and heart rate that causes variation in the Oxygen and Entonox treatment groups. Naturally, since oxygen is always required to be used when nitrous oxide is being given, this can only be speculation. 
Thanks to Jannsen Pharmaceuticals and the British Oxygen Company (Ohmeda) for their support in this research. Thanks to Mr D. G. Ferguson, Consultant in Accident and Emergency, Royal Hallamshire Hospital, Sheffield for allowing free access to his department, the patients and help from the junior staff. Thanks to Dr J. Johnston, MBChB for the cartoon pain scale.

\section{REFERENCES}

Dottori L., Korsgren M., Ax:son Lof B. \& Wilhelmsen L. (1976) The haemodynamic effects of unsupplemented nitrous oxide-oxygen relaxant anaesthesia in cardiac patients. Acta Anaesthesiologica Scandanavica 20, 195-200.

Dworkin S. F., Schubert M. M., Chen A. C. N. \& Clark D. W. (1983) Analgesic effects of nitrous oxide with controlled painful stimuli. American Journal of the Dental Association 107, 581-5.

Everett G. B. \& Allen G. D. (1971) Simultaneous evaluation of cardiorespiratory and analgesic effects of nitrous oxide-oxygen analgesia. Journal of the American Dental Association 83, 129-33.

Flomembaum N., Gallagher E. J., Eagen K. \& Jacobson S. (1979) Self administered nitrous oxide: an adjunct analgesic. Journal of the Amterican College of Entergency Physicians 8, 95-7.

Hallonsten A. L., Koch G. \& Schroder U. (1983) Nitrous oxide - oxygen sedation in dental care. Community Oral and Dental Epidemiology 11, 347-55.

Maloney J. M., Coleman W. P. \& Mora R. (1980) Analgesia induced by nitrous oxide and oxygen as an adjunct to local anaesthesia in dermatological surgery. Journal of Dermatological and Surgical Oncologe 6, 939-43.

McKinnon K. D. (1981) Pre-hospital analgesia with nitrous oxide/oxygen. Canadian Medical Associatia Journal 125, 836-40.

Melzack R. \& Torgerson W. S. (1971) On the language of pain. Anaesthesiology 34, 50-9.

Melzack R., Wall P. D. \& Ty T. C. (1982) Acute pain in an emergency clinic. Pain 14, 33-43.

Parbrook G. D. (1967) The levels of nitrous oxide analgesia. British Journal of Anaesthesia 1967; Vol 39: pp 974-981.

Parbrook G. D. \& Kennedy B. R. (1964) Value of pre-mixed nitrous oxide and oxygen in the relief of post operative pain. Double blind trial of $25 \%$ nitrous oxide in oxygen, $15 \%$ nitrous oxide in oxygen and oxygen. British Medical Journal 2, 1303-5.

Sonnerstein R. R. (1948) A study on the mechanism of nitrous oxide analgesia. Journal of Applied Physiology 1, 254.

Trieger N., Loskota W. J., Jacobs A. W. \& Newman M. G. (1974) Nitrous oxide - A study of the physiological and psychomotor effects. Journal of the American Dental Association 82, 142-50.

Unruh A., McGrath P., Cunninham S. J. \& Humphreys P., (1983) Children's drawings of their pain. Pain 17, 385-92. 